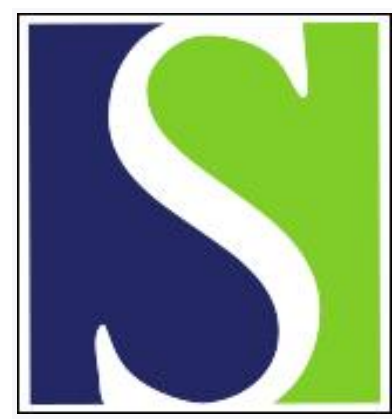

Scand J Work Environ Health 2011;37(1):30-36

https://doi.org/10.5271/sjweh.3121

Published online: 20 Sep 2010, Issue date: Jan 2011

Physical workload and accelerated occurrence of lumbar spine diseases: risk and rate advancement periods in a German multicenter case-control study

by Seidler A, Euler U, Bolm-Audorff U, Ellegast R, Grifka J, Haerting J, Jäger M, Michaelis M, Kuss $O$

Affiliation: Institute and Policlinic for Occupational and Social Medicine, Fetscherstrasse 74, D-01307 Dresden, Germany. andreas.seidler@mailbox.tu-dresden.de

The following articles refer to this text: 2013;39(1):5-26; 2013;39(5):486-494

Key terms: case-control study; EPILIFT; lumbar disc disease; lumbar spine disease; physical workload; RAP; risk acceleration; risk and rate advancement

This article in PubMed: www.ncbi.nlm.nih.gov/pubmed/20852832 


\title{
Physical workload and accelerated occurrence of lumbar spine diseases: risk and rate advancement periods in a German multicenter case-control study
}

\author{
by Andreas Seidler, MD, MPH, ${ }^{1}$ Ulrike Euler, MD, MSc, ${ }^{2}$ Ulrich Bolm-Audorff, MD, ${ }^{3}$ Rolf Ellegast, PhD, ${ }^{4}$ \\ Joachim Grifka, MD, ${ }^{5}$ Johannes Haerting, PhD, ${ }^{6}$ Matthias Jäger, PhD, ${ }^{7}$ Martina Michaelis, PhD, ${ }^{8}$ Oliver \\ Kuss, $P h D^{6}$
}

Seidler A, Euler U, Bolm-Audorff U, Ellegast R, Grifka J, Haerting J, Jäger M, Michaelis M, Kuss 0. Physical workload and accelerated occurrence of lumbar spine diseases: risk and rate advancement periods in a German multicenter case-control study. Scand J Work Environ Health. 2011;37(1):30-36.

\begin{abstract}
Objective In a German multicenter case-control study of lumbar disc diseases, we calculated risk and rate advancement periods (RAP) for physical workload.

Methods Patients aged 25-70 years with clinically and radiologically verified lumbar disc herniation (286 males, 278 females) or symptomatic lumbar disc narrowing (145 males, 206 females) were compared with population control subjects (453 males and 448 females). For this analysis, all manual handling of objects of about $\geq 5 \mathrm{~kg}$ and postures with trunk inclination of $\geq 20^{\circ}$, as assessed by technical experts, were included in the calculation of cumulative lumbar load, determined by biomechanical model calculations. Logistic regression analysis was applied to calculate RAP, adjusted for region.
\end{abstract}

Results We found a steep positive dose-response relationship between age and lumbar disc narrowing among men as well as women; however, we did not find a monotonic increase in lumbar disc herniation risk with age. As a monotonic increase in disease risk with age constitutes a fundamental assumption underlying the RAP concept, we restricted our RAP analysis to cases with symptomatic lumbar disc narrowing. Among men, there was a positive dose-response relationship between the cumulative lumbar load and the acceleration of lumbar disc narrowing. In the highest exposure category, a RAP of 28.0 years [ $95 \%$ confidence interval $(95 \% \mathrm{CI}) 9.7-46.3$ years] was found. Among women, the RAP was 8.8 years (95\% CI 2.4-15.2 years) in the highest exposure category.

Conclusion This study emphasizes the conceptual importance of risk acceleration - causation not only comprises the occurrence of a disease (that without a specific exposure would not have occurred at all) but also the accelerated occurrence of a disease (that without exposure would have occurred later in life).

Key terms: EPILIFT; lumbar disc disease; RAP; risk acceleration.

For the recognition of occupational diseases, compensation boards and courts often have to clarify if the causation of a disease through a specific occupational exposure is more likely than not. This requirement is mostly "translated" in epidemiologic terms to the question of whether the probability of causation for exposed individuals exceeds 50\% [the "more-likely-than-not" rule, see (1)]. If the attributable risk estimated from epidemiologic data is equated to the probability of causation, the so-called "doubling dose" (meaning the exposure level at which the relative risk equals two) has to be exceeded to assume an occupational causation of disease. Greenland (1) has pointed to a "key fallacy" in the equation of probability of causation and attributable risk - causation not only comprises the occurrence of a disease (that without exposure would not have occurred at all) but also the accelerated

1 Institute and Policlinic for Occupational and Social Medicine, Technical University Dresden, Dresden, Germany

2 Federal Institute for Occupational Safety and Health, Berlin, Germany

3 Labor inspection, Occupational Health Division, Regional Government of South Hesse, Wiesbaden, Germany

4 Institute for Occupational Safety and Health of the German Social Accident Insurance (IFA), Sankt Augustin, Germany

5 Orthopaedic Clinic, University of Regensburg, Bad Abbach, Germany

6 Institute for Medical Epidemiology, Biostatistics, and Informatics, Martin-Luther-University Halle-Wittenberg, Halle (Saale), Germany

7 Leibniz Research Centre for Working Environment \& Human Factors, Dortmund University of Technology, Dortmund, Germany

8 Research Center for Occupational and Social Medicine Freiburg, Freiburg, Germany

Correspondence to: Prof. Dr. Andreas Seidler, Institute and Policlinic for Occupational and Social Medicine, Fetscherstrasse 74, D-01307 Dresden, Germany. [E-mail: andreas.seidler@mailbox.tu-dresden.de] 
occurrence of a disease (that without exposure would have occurred later in life). As the attributable risk calculation is predominantly based on the excess risk, the established relative risk estimators tend to underestimate the probability of causation. The resulting error might be particularly pronounced in frequent chronic diseases with clear age dependency.

In 1993, Brenner et al (2) proposed an effect estimator that accounts for the acceleration of disease occurrence, the so-called "risk and rate advancement periods" (RAP). The basic principle of RAP is to estimate the increase of risk with age: RAP are the time periods by which the risk or rate of disease is accelerated among exposed subjects conditional on disease-free survival to some baseline age (2). Since its first publication, RAP estimation has been applied, for example, to assess premature myocardial infarction risks through smoking, hypertension, and dyslipidemia (3) and to estimate the acceleration of mortality through traffic air pollution (4) and the impact of manual material handling on low-back pain (5).

We calculated RAP from a case-control study of lumbar disc diseases [EPILIFT (6)]. The aim of this analysis was firstly to describe the potential impact of physical workload on lumbar disc diseases in terms of risk acceleration and, secondly, to further elucidate the suitability of RAP to express causality for social insurance (recognition of occupational diseases) and preventive purposes.

\section{Methods}

\section{Study participants}

The design of the German EPILIFT study has been described in detail in a previous publication (6). Briefly, in four study regions (Frankfurt am Main, Freiburg, Halle/Saale, Regensburg) all hospitals or practices treating $\geq 5$ patients with lumbar disc herniation per year $(\mathrm{N}=29)$ as well as a random sample of orthopedic practices $(\mathrm{N}=14)$ were included. The corresponding physicians were asked to identify all patients between 25-70 years of age that belonged to one of the following case groups: males (case group 1, $\mathrm{N}=286$ ) or females (case group 2, N=278) treated due to lumbar disc herniation with radiculopathy with sensory and/or motor deficits; males (case group 3, $\mathrm{N}=145$ ) or females (case group $4, \mathrm{~N}=206$ ) treated due to severe disc narrowing with radiculopathy with sensory and/or motor deficits or with local lumbar syndrome with a finger floor distance of $>25 \mathrm{~cm}$. Recruiting physicians had to state the date of initial radiographic diagnosis of lumbar disc disease; patients were not eligible for the study if the initial diagnosis of lumbar disc disease had been made $>10$ years earlier. The diagnosis of lumbar disc herniation had to have been confirmed by a reference radiologist by either computerized tomography or by magnetic resonance imaging (MRI); the diagnosis of lumbar disc narrowing was primarily based on X-ray. Disc herniation and narrowing were defined as proposed by a German consensus group (7). The clinical diagnosis had to be verified by one experienced reference orthopedist based on standardized findings sheets. The participation rate was $66.4 \%$ among the cases. The response rate of cases was 58.8\% in Frankfurt am Main, 61.8\% in Freiburg, 77.2\% in Halle and $70.4 \%$ in Regensburg. The response rate did not differ between male and female cases.

Control subjects were randomly selected from a $1 \%$ random sample of residents aged $25-70$ years drawn by the local population registration offices of the respective region. The participation rate was $53.4 \%$ among the control subjects. The response rate of control subjects was $50.8 \%$ in Frankfurt am Main, $46.1 \%$ in Freiburg, $61.6 \%$ in Halle and $58.4 \%$ in Regensburg. As in cases, the response rate did not differ between male and female control subjects. Due to lack of radiographic examination, the frequency of lumbar disc disease is unknown among the population controls. A suspected prevalence of lumbar disc disease of up to $10 \%$ among population controls would result in a slight tendency to underestimate potential risk factors.

As the low participation rate might have introduced a selection bias, we asked non-participants by telephone about their current job: $57 \%$ of non-participating cases and $47 \%$ of non-participating control subjects gave their current occupation. According to this information, the proportion of blue-collar workers was higher among non-participating cases $(62 \%$ among non-participants, $50 \%$ among participants) and control subjects (50\% among non-participants, 37\% among participants). Taking into account the higher response rate among cases, the responder analysis does not point to a substantial differential selection bias.

\section{Ethics}

This study was performed in compliance with the ethical principles of the Helsinki Declaration. All subjects gave their informed consent. The Hesse State Chamber of Physicians controlled the study protocol of EPILIFT. The ethics commission formulated no ethical or legal concerns against the conduction of the EPILIFT study given that the Hesse Federal Commissioner for Data Protection and Freedom of Information Decline had no concerns. In a second step, the Hesse Federal Commissioner for Data Protection and Freedom of Information Decline conducted another personal hearing and gave some comments, which we considered in our informed consent. After these requirements were fulfilled, the Hesse Data 
protection officer had no further concerns. The authors declare they have no competing interests.

\section{Exposure assessment}

Cumulative physical workload (expressed by cumulative lumbar load) was assessed in a two-step procedure. First, a standardized computer-assisted interview was applied to identify subjects that exceeded certain minimum workloads through lifting and carrying of objects, pushing and pulling, working in trunk-flexed postures, and whole-body vibration. Second, a comprehensive semistandardized personal expert interview was performed with those subjects who (on the basis of the standardized personal interview) exceeded those relatively low "exposure thresholds" (63.3\% of study participants). These interviews were conducted by branch-orientated ergonomic experts of the institutions for statutory accident insurance and prevention. On the basis of job-task specific supplementary surveys, these experts documented (blind for the case-control status) the intensity, frequency, and duration of all spine-related exposures induced by manual materials handling (eg, lifting, carrying, pushing, pulling, throwing, catching, or shoveling of all objects weighing $\geq 5 \mathrm{~kg}$ ), trunk inclination and twisting, and whole-body vibration for the entire working life of each subject (8). A biomechanical analysis based on this expert assessment was conducted to determine the compressive force on the lumbosacral disc for each documented loading activity (range $0-8.4 \mathrm{kN}$ ). Quantification was based on biomechanical-model calculations applying the three-dimensional dynamic simulation tool "The Dortmunder" (9).

Several cumulative dose models (10) - each of them based on disc-compressive force, duration, and frequency of occurrence, but varying in thresholds at calculating the lifetime lumbar dose - were considered (and further dose models are currently considered in an in-depth analysis) to describe the best dose-response relationship between lifetime lumbar load and lumbar disc diseases. However, the question of the "best" dose model is of minor importance to the question of the suitability of the RAP as effect measures. Therefore, just as in the cited previous publication (6), in this analysis all biomechanically relevant loading actions that are assumed to potentially contribute to the development of lumbar disc disease are included in the calculation of cumulative lumbar load (ie, for the calculation of cumulative lumbar load manual handling of objects of about $\geq 5 \mathrm{~kg}$ and postures with trunk inclination of $\geq 20^{\circ}$ are considered). Lumbar disc compressive force is weighted overproportionally (squared) in relation to the respective duration of materials handling or intensive-load posture. Generally, among cases only exposures up to the date of first diagnosis were considered for analysis.

\section{Statistical analysis}

Logistic regression analysis was applied to calculate RAP, which describe how much sooner a given risk of lumbar spine disease occurs among exposed than unexposed subjects. In the calculation of RAP, we applied the same categorization procedure of variables as reported by Seidler et al (6). For computation of a RAP, age has to be included as a continuous variable in the regression model. Region (place of residence) was considered to be a potential confounder because occupational exposures were suspected to differ between regions. Applying an a-priori-defined procedure for the selection of potential confounders as explained by Seidler et al (6), we found that lifestyle factors did not confound the risk estimators for physical workload. Therefore, lifestyle was not included as a confounder in the regression models. Notwithstanding our final model for case group 1 (males with lumbar disc herniation) reported by Seidler et al (6), we did not adjust for distress by unemployment, as we cannot exclude that this factor might be influenced by physical workload (potentially introducing "cause and effect" bias). Socioeconomic status is itself likely to influence the choice of occupation towards physically demanding jobs. Additional adjustment for socioeconomic status might consequently lead to an underestimation of the relation between physical workload and lumbar spine disease. We therefore decided not to control for socioeconomic status. Consequently, in the calculation of RAP, only region (males) and region and psychosocial workload (females) are included as confounders in the logistic regression models.

A fundamental assumption underlying the RAP concept is a monotonic increase in disease risk with age. To verify this assumption, we analyzed the relationship between age and lumbar spine disease risk. First we calculated odds ratios (OR) for age in 10-year categories $(<35$ years as reference; $35-<45$ years; $45-<55$ years; $55-<65$ years; $\geq 65$ years). If the results of this categorized analysis pointed to a monotonic increase in disease risk with age, we further analyzed the relationship between age as a continuous variable and the diagnosis of lumbar disc disease. We included age as a linear variable, additionally as a quadratic term $\left(\mathrm{age}^{2}\right)$ and as a cubic term $\left(\mathrm{age}^{3}\right)$. To decide about the adequate form in which age should enter the model, we used the Akaike information criterion (AIC).

Assuming a linear age term to reflect adequately the relationship between age and lumbar spine disease, we useD the lumbar spine disease risk for subjects with a specific (categorized) physical workload compared with non-exposed subjects (regression coefficient $b_{l}$ ) and the lumbar spine disease risk for each year of age (regression coefficients $b_{2}$ ) to estimate the RAP. A point estimate of the RAP is given by the ratio of the regres- 
sion coefficients $b_{1}$ and $b_{2}\left(\mathrm{RAP}=b_{1} / b_{2}\right)$. We calculated $95 \%$ confidence intervals $(95 \% \mathrm{CI})$ applying the delta method as described by Brenner et al (2). In case of a more complex dose-response relationship between age and lumbar spine disease risk, the RAP would vary with age: if, for example, the dose-response relationship between age and lumbar spine disease risk would be best expressed by a positive quadratic age term, then the RAP would decrease with increase in age.

Considering the interaction term between age and physical workload (results not shown), in our study, there was no indication for a relevant aberration from a multiplicative interaction mode. We therefore did not perform any further interaction analyses.

\section{Results}

The relationship between age and lumbar spine disease risk is shown in table 1. As a result of this analysis, we did not find a monotonic increase in lumbar disc herniation risk with age, but we found a steep positive dose-response relationship between age and lumbar disc narrowing among men as well as women. Compared to individuals $<35$ years of age, men aged $>65$ years had an OR for lumbar disc narrowing of 4.1 (95\% CI 1.7-9.6); women had an OR of 28.2 (95\% 9.0-87.8). However, the point estimators have to be interpreted cautiously because of the small case numbers in the reference groups (ie, $<35$ years old subjects). As the mentioned fundamental assumption is fulfilled only for lumbar disc narrowing, the following analyses are restricted to this disease.

To investigate the dose-response-relationship between age and lumbar disc narrowing, we first included age as a linear variable in the logistic regression model, revealing a highly significant association between age and diagnosis of lumbar disc narrowing $(\mathrm{P}<0.0005)$. If we additionally included age as a quadratic or even cubic term, these additional terms were not significantly associated with the diagnosis of lumbar disc narrowing. Furthermore, the inclusion of a quadratic or cubic age term did not improve the goodness-of-fit of the regression model as indicated by AIC. Therefore, we included age as a linear variable in the logistic regression model.

RAP are given in table 2. Among men, there was a positive dose-response relationship between the cumulative lumbar load (through manual materials handling and/or intensive-load postures) and the acceleration of lumbar disc narrowing. In the middle exposure group, the RAP of the final model was non-significantly elevated to 10.2 years $(95 \% \mathrm{CI}-4.0-24.4$ years), in the highest exposure category, it was significantly elevated to 28.0 years ( $95 \%$ CI 9.7-46.3 years). This means that, for example, a 40 -year-old man with a high cumulative physical workload has a certain risk of symptomatic lumbar disc narrowing that he would be expected to have had at age 68 (28 years later) in case he had not had any physical workplace exposure of the assumed intensity. Among women, the RAP were less pronounced: the RAP of the final model was 10.8 years $(95 \%$ CI $4.2-17.4$ years) in the second-highest exposure category; in the highest exposure category, the RAP did not further increase ( $\mathrm{RAP}=8.8,95 \%$ CI 2.4-15.2 years).

\section{Discussion}

In this study, we found a monotonic increase with age in lumbar disc narrowing, but not in lumbar disc herniation. This finding is in accordance with the literature. Applying computerized tomography, Kalichman et al (11) revealed a clear age-dependency of disc narrowing. While in their study, $25 \%$ of subjects aged $<40$ years showed disc space narrowing, the prevalence rose to $55.3 \%$ among $40-49$ -

Table 1. Age (10-year categories) and lumbar disc disease risk

\begin{tabular}{|c|c|c|c|c|c|c|c|c|c|c|c|c|c|c|c|c|}
\hline \multirow[t]{2}{*}{ Age at diagnosis/interview } & \multicolumn{4}{|c|}{ Control subjects } & \multicolumn{6}{|c|}{ Lumbar disc herniation } & \multicolumn{6}{|c|}{ Lumbar disc narrowing } \\
\hline & Mean & SD & $\mathrm{N}$ & $\%$ & Mean & SD & N & $\%$ & $O R^{a}$ & $95 \% \mathrm{Cl}$ & Mean & SD & N & $\%$ & $\mathrm{OR}^{\mathrm{a}}$ & $95 \% \mathrm{Cl}$ \\
\hline Men & 47.3 & 12.6 & & & 48.7 & 11.1 & & & & & 55.0 & 10.7 & & & & \\
\hline$<35$ years & & & 84 & 18.5 & & & 27 & 9.4 & 1.0 & & & & 9 & 6.2 & 1.0 & \\
\hline $35-<45$ years & & & 119 & 26.3 & & & 91 & 31.8 & 2.1 & $1.3-3.7$ & & & 20 & 13.8 & 1.2 & $0.5-2.9$ \\
\hline $45-<55$ years & & & 97 & 21.4 & & & 73 & 25.5 & 1.9 & $1.1-3.4$ & & & 31 & 21.4 & 2.1 & $0.9-4.9$ \\
\hline $55-<65$ years & & & 104 & 23.0 & & & 61 & 21.3 & 1.3 & $0.8-2.4$ & & & 51 & 35.2 & 2.9 & $1.3-6.6$ \\
\hline$\geq 65$ years & & & 49 & 10.8 & & & 34 & 11.9 & 1.4 & $0.7-2.7$ & & & 34 & 23.4 & 4.1 & $1.7-9.6$ \\
\hline Women & 46.4 & 11.8 & & & 47.1 & 11.8 & & & & & 56.0 & 9.8 & & & & \\
\hline$<35$ years & & & 77 & 17.2 & & & 51 & 18.3 & 1.0 & .. & & & 4 & 1.9 & 1.0 & \\
\hline $35-<45$ years & & & 137 & 30.6 & & & 71 & 25.5 & 0.7 & $0.4-1.1$ & & & 27 & 13.1 & 3.2 & $1.1-9.6$ \\
\hline $45-<55$ years & & & 109 & 24.3 & & & 72 & 25.9 & 0.9 & $0.6-1.5$ & & & 54 & 26.2 & 8.0 & $2.7-23.7$ \\
\hline $55-<65$ years & & & 94 & 21.0 & & & 58 & 20.9 & 0.9 & $0.5-1.5$ & & & 78 & 37.9 & 14.6 & $5.0-42.8$ \\
\hline$\geq 65$ years & & & 31 & 6.9 & & & 26 & 9.4 & 1.4 & $0.7-2.6$ & & & 43 & 20.9 & 28.2 & $9.0-87.8$ \\
\hline
\end{tabular}

a Adjusted for region. 
Table 2. Odds ratios (OR) and risk and rate advancement periods (RAP) of symptomatic lumbar disc narrowing with cumulative lumbar load through manual materials handling and/or intensive-load postures. [95\% Cl=95\% confidence interval.]

\begin{tabular}{|c|c|c|c|c|c|c|c|c|c|}
\hline \multirow[b]{3}{*}{ Men } & \multicolumn{2}{|c|}{ Cases } & \multicolumn{2}{|c|}{$\begin{array}{l}\text { Control } \\
\text { subjects }\end{array}$} & \multirow[t]{2}{*}{$\mathrm{OR}^{\mathrm{a}}$} & \multirow[t]{2}{*}{$95 \% \mathrm{Cl}$} & \multirow[t]{2}{*}{$\begin{array}{l}\text { Regression } \\
\text { coefficient }\end{array}$} & \multirow[t]{2}{*}{$\begin{array}{l}\text { RAP } \\
\text { (years) }\end{array}$} & \multirow[t]{2}{*}{$95 \% \mathrm{Cl}$} \\
\hline & N & $\%$ & N & $\%$ & & & & & \\
\hline & & & & & & & & & \\
\hline Age & & & & & 1.043 & $1.024-1.062$ & 0.042 & & \\
\hline \multicolumn{10}{|c|}{$\begin{array}{l}\text { Cumulative lumbar load through manual materials handling } \\
\text { and/or intensive-load postures }\end{array}$} \\
\hline $0-<5.0 \times 10^{6} \mathrm{Nh}$ & 27 & 18.6 & 159 & 35.1 & 1.0 & .. & & 0 & \\
\hline $5.0-<21.51 \times 10^{6} \mathrm{Nh}$ & 31 & 21.4 & 147 & 32.5 & 1.5 & $0.9-2.8$ & 0.429 & 10.2 & $-4.0-24.4$ \\
\hline$\geq 21.51 \times 10^{6} \mathrm{Nh}$ & 87 & 60.0 & 147 & 32.5 & 3.1 & $1.8-5.2$ & 1.121 & 28.0 & $9.7-46.3$ \\
\hline \multicolumn{10}{|l|}{ Women } \\
\hline Age & & & & & 1.083 & $1.064-1.104$ & 0.080 & & \\
\hline $\begin{array}{l}\text { Cumulative lumbar loa } \\
\text { and/or intensive-load } p\end{array}$ & & & & & & & & & \\
\hline $\mathrm{ONh}$ & 55 & 26.7 & 195 & 43.5 & 1.0 & .. & & 0 & .. \\
\hline$>0-<4.04 \times 10^{6} \mathrm{Nh}$ & 28 & 13.6 & 84 & 18.8 & 1.2 & $0.7-2.2$ & 0.212 & 2.7 & $-10.7-16.1$ \\
\hline $4.04-<14.47 \times 10^{6} \mathrm{Nh}$ & 50 & 24.3 & 85 & 19.0 & 2.4 & $1.4-4.1$ & 0.867 & 10.8 & 4.2-17.4 \\
\hline$\geq 14.47 \times 10^{6} \mathrm{Nh}$ & 73 & 35.4 & 84 & 18.8 & 2.0 & $1.2-3.3$ & 0.701 & 8.8 & $2.4-15.2$ \\
\hline
\end{tabular}

${ }^{a}$ Adjusted for region (males) or region and psychosocial workload (females).

year old subjects, to $70.3 \%$ among $50-59$-year olds, and to $83.3 \%$ among those $>60$ years. However, we cannot exclude differences in clinical practice according to the age of patients. If, for example, patients with lumbar spine diseases were more likely to be investigated radiographically at older ages, this might spuriously exaggerate the age-dependency of symptomatic lumbar disc narrowing.

Several studies support our finding of an inversely U-shaped age dependency of lumbar disc herniation. Brüske-Hohlfeld et al (12) found the highest age-specific incidence of first lumbar disc operation for suspected prolapsed in middle-aged persons (mean age 42 years). Dammers \& Koehler (13) also found an age peak among middle-aged patients suffering from lumbar disc herniation (mean age 44.1 years) at the lowest spinal segment (L5-S1), however, with increasing herniation levels, the mean age increased. As the non-monotonic age-dependency of lumbar disc herniation risk makes the calculation of RAP impossible, the following discussion is restricted to lumbar disc narrowing.

Among our patients with symptomatic lumbar disc narrowing, radiographic findings (lumbar disc narrowing) might partly be incidentally associated with low-back pain. This would mean that, among these patients, lowback pain would not be attributable to the narrowing. However, our case definition "symptomatic lumbar disc narrowing" does not rely just on the existence of low back pain. Of male cases with symptomatic lumbar disc narrowing, 31\% had a radiculopathy with motor or sensomotor deficits corresponding with the localization of the narrowed discs, $23 \%$ had a corresponding radiculopathy with sensory deficits, and $46 \%$ solely had a local lumbar syndrome with a finger floor distance of $>25 \mathrm{~cm}$. Of female cases with symptomatic lumbar disc narrowing, $25 \%$ had a radiculopathy with motor or sensomotor deficits corresponding with the localization of the narrowed discs, 19\% had a radiculopathy with sensory deficits, and $54 \%$ solely had a local lumbar syndrome with a finger floor distance of $>25 \mathrm{~cm}$. Altogether, in about half of our cases with symptomatic lumbar disc narrowing, the radiographic signs correspond to the neurological finding of a radiculopathy with motor or sensory deficits. Moreover, with low-back-pain-free control subjects as reference, OR for physically exposed cases with symptomatic lumbar disc narrowing were considerably higher than those of physically exposed control subjects with low-back pain (14). Therefore, our cases with symptomatic lumbar disc narrowing do not just reflect cases with low-back pain.

We found an adjusted RAP for symptomatic lumbar disc narrowing of 28 years for men and close to 9 years for women in the highest cumulative physical workload category. As the point estimators of the relative risk (as indicated by OR) do not substantially differ between men and women in our study, the gender-specific RAP differences cannot be explained by differences in relative risks for physical workload. In fact the relatively weak age effect among men is responsible for the relatively high RAP: the magnitude of the RAP is by definition inversely related to the strength of the age effect.

To our knowledge, no conclusive data are available in the literature that allow an in-depth comparison of the age-dependent prevalence of lumbar disc narrowing between men and women. Among a representative sample of 40 -year-old Danish subjects ( $\mathrm{N}=412$ ), $59 \%$ of men but only $48 \%$ of women had a reduced lumbar disc height as diagnosed by MRI (15). The finding of a lower disc narrowing prevalence of middle-aged women in comparison with middle-aged men is in accordance with Kauppila et al (16) who found, in a subsample of the Framingham cohort, $19 \%$ of the 54 -year-old (on average) men but only $12 \%$ of the women showed disc space narrowing in the conventional lumbar radiograph. In contrast, at the 
follow-up examination about 25 years later, $60 \%$ of both participating men and women showed disc space narrowing. This result would be compatible with our finding of a stronger age effect (concerning disc space narrowing) among women than men. However, it has to be pointed out that the sparse data allowing gender-specific comparisons are inconsistent. While, for example, the mentioned studies of Kjaer et al (15) and Kauppila et al (16) found a lower prevalence of disc space narrowing among middleaged women, Kalichman et al (17) found a comparable prevalence of disc space narrowing among 53-years-old (on average) men and women (64.1\% of men, $63.8 \%$ of women) applying computerized tomography.

Apart from possible (to date unknown) biologic reasons for gender-specific differences in the age effect of lumbar disc narrowing, methodological issues have to be taken into account as a potential explanation for the differences in the age effect between men and women. In casecontrol studies, age-specific risk estimators and therefore also RAP point estimators are sensitive to age-specific differences in participation between cases and control subjects. While, among control subjects, we observed the highest response rate among 55-65 year-old men (66\%) and women $(68 \%)$, the response rate was, for example, notably lower among women aged $>65$ years $(39 \%)$ than men $>65$ years $(60 \%)$. Among women, the "crude" risk increase per year of age ( $7.5 \%$, solely adjusted for region) remained nearly unchanged when non-responding control subjects were included in the control group (7.3\%). However, among men the "crude" risk increase per year of age slightly rose from $5.3 \%$ to $6.0 \%$ when non-responding control subjects were included in the control group. Therefore, age-specific differences in the response rate between men and women might partly explain the different RAP. The weaker the strength of the age effect, the higher the RAP point estimators, which limits their informative value. Against the background of the hitherto sparse experience with RAP, RAP point estimators should be interpreted with particular caution in case of a weak age effect.

Several authors highlight the improved risk communication provoked by estimators of the acceleration of disease occurrence in comparison with estimators of relative risk $(3,5)$. In this respect, a better understanding of potential risks in the occupational health field might more effectively motivate decision-makers to enhance preventive measures. In clinical epidemiology, several studies have dealt with the dependency of data interpretation on the format of effect estimation, particularly questioning the appropriateness of relative risk estimators for risk communication (18). We cannot be sure at all that RAP estimators really lead to a better understanding of potential risks - for example, a RAP of 28 years (as found in our study) could just as well lead to a substantial overestimation of the benefits to be expected by reductions of exposure. One has to keep in mind that the RAP concept does not totally differ from that of relative risk; in fact, the RAP point estimator is just the quotient of two (logarithmized) relative risk estimators. Therefore, the better appropriateness of RAP (as an example for an estimator of the acceleration of disease occurrence) still has to be empirically substantiated.

Another aspect requires a careful interpretation of estimators of the acceleration of disease occurrence. When RAP are used to allow for the acceleration of disease occurrence as a causal effect, this suggests a specific etiologic mechanism: without the exposure under consideration, the disease would also have occurred, but at a later time. If an occupational exposure (for instance, a toxic substance) led, among all exposed individuals, to a generally age-dependent disease (for instance, acute renal failure), predominantly individuals who, without the exposure would not have developed this disease, would be expected to develop acute renal failure; Greenland calls this mechanism "unaffected occurrence" (1). In this case, the calculation of a RAP would be feasible, but would not make sense. Therefore, the RAP should only be calculated when exposed persons are expected to develop a disease that would have occurred, without the exposure, at a later time, what Greenland refers to as "accelerated occurrence" (1). Lumbar disc narrowing is a chronic "progressive" process that sooner or later is believed to affect all or at least most persons. Therefore, it appears biologically plausible, that a high proportion of lumbar spine narrowing caused by physical workload is due to accelerated occurrence. Nevertheless epidemiologic data cannot distinguish accelerated occurrences from unaffected occurrences (1). Greenland states that "the only way one can estimate the relative proportion of accelerated and unaffected occurrences - and hence estimate the probability of causation - is by positing a specific biologic model for the disease process" (1). Therefore, even when epidemiologic estimators of the acceleration of disease occurrence (for example, RAP) can be useful to illustrate the exposure-dependence of timing of disease occurrence, statistical methods alone cannot decide about the reasonability of the acceleration concept. In-depth pathophysiologic knowledge is needed next to in-depth epidemiologic knowledge to adequately describe the causal relationship between exposure and disease occurrence.

\section{Concluding remarks}

In this population-based case-control study, RAP are calculated to describe how much sooner a given risk of symptomatic lumbar spine narrowing occurs among subjects with heavy physical workload. We find RAP of $\leq 28$ years (among men) which might partly be explained by age-specific differences in participation between cases and control subjects. Independent from methodological considerations, the authors would like to emphasize the 
conceptual importance of risk acceleration - causation not only comprises the occurrence of a disease (that without exposure would not have occurred at all) but also the accelerated occurrence of a disease (that without exposure would have occurred later in life).

\section{Acknowledgements}

This work was supported by a grant from the German Social Accident Insurance (DGUV). We would like to thank the following persons for their valuable contributions: Dr Annekatrin Bergmann, Dirk Ditchen, Professor Dr Gine Elsner, Dr Siegfried Fischer, Oliver Geiß, Dr Cornelia Handschuh, Dr Stefan Hensler, Professor Dr Friedrich Hofmann, Andrea Kamerewerd, Andrea Kronen, PD, Dr Oliver Linhardt, Karin Lukaszewski, Professor Dr Alwin Luttmann, Yvonne Martin, Ilona Meurer-Wurzer, Dr Matthias Nübling, Dr Petereit-Haack, Barbara Piehler, Thorsten Roth, Christoph Schiefer, Dr Barbara Schumann, Dr Regina Skölziger, Dr Karen Spannhake, Gabriele Volante, and Jürgen Voß.

\section{References}

1. Greenland S. Relation of probability of causation to relative risk and doubling dose: a methodologic error that has become a social problem. Am J Public Health. 1999;89:1166-69.

2. Brenner H, Gefeller O, Greenland S. Risk and rate advancement periods as measures of exposure impact on the occurrence of chronic diseases. Epidemiology. 1993;4:229-236.

3. Liese AD, Hense HW, Brenner H, Löwel H, Keil U. Assessing the impact of classical risk factors on myocardial infarction by rate advancement periods. Am J Epidemiol. 2000;152:884-8.

4. Finkelstein MM, Jerrett M, Sears MR. Traffic air pollution and mortality rate advancement periods. Am J Epidemiol. 2004;160:173-177.

5. Latza U, Pfahlberg A, Gefeller O. Impact of repetitive manual materials handling and psychosocial work factors on the future prevalence of chronic low-back pain among construction workers. Scand J Work Environ Health. 2002;28:314-23.

6. Seidler A, Bergmann A, Jäger M, Ellegast R, Ditchen D, Elsner $\mathrm{G}$, et al. Cumulative occupational lumbar load and lumbar disc disease - results of a German multi-center case-control study (EPILIFT). BMC Musculoskelet Disord. 2009;7:10-48.

7. Bolm-Audorff U, Brandenburg S, Brüning T, Dupuis H, Ellegast $\mathrm{R}$, Elsner G, et al. Medizinische Beurteilungskriterien zu bandscheibenbedingten Berufskrankheiten der Lendenwirbelsäule (I) Konsensempfehlungen zur Zusammenhangsbegutachtung der auf Anregung des HVBG eingerichteten interdisziplinären Arbeitsgruppe. Trauma und Berufskrankheit [Medical evaluation criteria for occupational diseases of the lumbar spine related to the intervertebral disc. Consensus recommendations for assessing the interrelationship as proposed by the interdisciplinary working group established by the HVBG]. Trauma und Berufskrankheit 2005;7:211-252.

8. Ellegast R, Ditchen D, Bergmann A, Bolm-Audorff U, Elsner G, Grifka J, et al. Erhebungen zur beruflichen Wirbelsäulenexposition durch die Technischen Aufsichtsdienste der Unfallversicherungsträger im Rahmen der Deutschen Wirbelsäulenstudie [Survey on work-related spinal exposures by technical experts of the German accident insurance institutions within the German Spine Study]. Zbl Arbeitsmed. 2007;57:251-263.

9. Jäger M, Luttmann A, Göllner R, Laurig W. The Dortmunder - Biomechanical model for quantification and assessment of the load on the lumbar spine. Technical paper presented at Digital Human Modeling For Design And Engineering Conference And Exhibition in June 2001, Arlington, VA, USA. Edited by: Soc Autom Eng. Arlington (VA):SAE, 2001.

10. Jäger M, Geiß O, Bergmann A, Bolm-Audorff U, Ditchen $\mathrm{D}$, Ellegast R, et al. Biomechanische Analysen zur Belastung der Lendenwirbelsäule innerhalb der Deutschen Wirbelsäulenstudie [Biomechanical analyses on lumbar load within the German Spine Study]. Zbl Arbeitsmed. 2007;57:264-276.

11. Kalichman L, Kim DH, Li L, Guermazi A, Hunter DJ. Computed tomography - evaluated features of spinal degeneration: prevalence, intercorrelation, and association with self-reported low back pain. Spine J. 2010;10:200-8.

12. Brüske-Hohlfeld I, Merritt JL, Onofrio BM, Stonnington $\mathrm{HH}$, Offord KP, Bergstralh EJ, et al. Incidence of lumbar disc surgery. A population-based study in Olmsted County, Minnesota, 1950-1979. Spine. 1990;15:31-35.

13. Dammers R, Koehler PJ. Lumbar disc herniation: level increases with age. Surg Neurol. 2002;58:209-13.

14. Seidler A, Bolm-Audorff U, Bergmann A, Ditchen D, Ellegast R, Elsner G, et al. Deutsche Wirbelsäulenstudie: Unterscheiden sich Chondrose-Risiken von Risiken für Low-Back-Pain? [German Lumbar Spine Study: Do chondrosis risks differ from low back pain-risks?] In: Letzel S (Ed.) Proceedings from the 47 Jahrestagung der Deutschen Gesellschaft für Arbeits und Umweltmedizin [47th Annual Meeting of the German Society for Occupational and Environmental Medicine] on 21-24 March 2007 in Mainz. Stuttgart: Gentner-Verlag 2007.

15. Kjaer P, Leboeuf-Yde C, Korsholm L, Sorensen JS, Bendix T. Magnetic resonance imaging and low back pain in adults: a diagnostic imaging study of 40-year-old men and women. Spine. 2005;30:1173-80.

16. Kauppila LI, McAlindon T, Evans S, Wilson P, Kiel D, Felson DT. Disc degeneration/back pain and calcification of the abdominal aorta: a 25-year follow-up study in Framingham. Spine. 1997;22:1642-47.

17. Kalichman L, Li L, Guermazi A, Hunter DJ. Association between age, sex, BMI and CT-evaluated spinal degeneration features. J Back Musculoskeletal Rehabil. 2009;27:189-95.

18. Covey J. A meta-analysis of the effects of presenting treatment benefits in different formats. Med Decis Making. 2007;27:638

Received for publication: 10 June 2010 